Acta Crystallographica Section D

\title{
Biological
}

Crystallography

ISSN 0907-4449

Editors: E. N. Baker and Z. Dauter

\section{Charge-density study on cyclosporine A}

\author{
S. K. J. Johnas, B. Dittrich, A. Meents, M. Messerschmidt and E. F. Weckert
}

Acta Cryst. (2009). D65, 284-293

Copyright (C) International Union of Crystallography

Author(s) of this paper may load this reprint on their own web site or institutional repository provided that this cover page is retained. Republication of this article or its storage in electronic databases other than as specified above is not permitted without prior permission in writing from the IUCr.

For further information see http://journals.iucr.org/services/authorrights.html

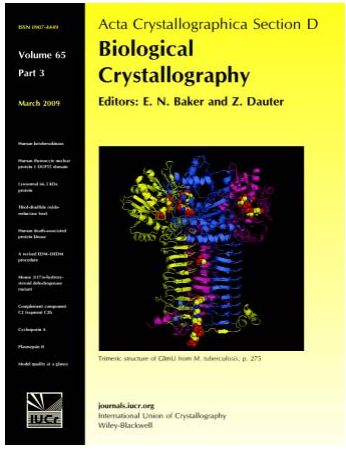

Acta Crystallographica Section D: Biological Crystallography welcomes the submission of papers covering any aspect of structural biology, with a particular emphasis on the structures of biological macromolecules and the methods used to determine them. Reports on new protein structures are particularly encouraged, as are structure-function papers that could include crystallographic binding studies, or structural analysis of mutants or other modified forms of a known protein structure. The key criterion is that such papers should present new insights into biology, chemistry or structure. Papers on crystallographic methods should be oriented towards biological crystallography, and may include new approaches to any aspect of structure determination or analysis.

Crystallography Journals Online is available from journals.iucr.org 
Acta Crystallographica Section D

Biological

Crystallography

ISSN 0907-4449

S. K. J. Johnas, ${ }^{a *}$ B. Dittrich, A. Meents, ${ }^{a}$ M. Messerschmidt ${ }^{a}$ and E. F. Weckert ${ }^{\mathrm{a} *}$

a HASYLAB at DESY, Notkestrasse 85, D-22607 Hamburg, Germany, and ${ }^{\mathbf{b}}$ Institut für Anorganische Chemie der Universität Göttingen, Tammannstrasse 4, D-37077 Göttingen, Germany

Correspondence e-mail: simone.johnas@desy.de, edgar.weckert@desy.de

\section{Charge-density study on cyclosporine A}

Two single-crystal X-ray diffraction data sets of cyclosporine A were measured to high resolution using synchrotron radiation at temperatures of 5 and $90 \mathrm{~K}$. They allowed an accurate determination of its molecular and electronic structure. Three electron-density models based on pseudoatom scattering factors were compared in terms of derived bond topological properties and in terms of electron-density differences on a grid. In one model multipole parameters were freely refined, whereas in the other two models the density was built up from fixed database parameters from the invariom database and University at Buffalo Databank. The data quality not only allowed benchmarking of the quality of both databases with the refined density, but also judgement of the feasibility of a multipole refinement of a larger oligopeptide structure such as cyclosporine A. Both databases performed equally well and reproduced the experimentally determined charge density satisfactorily.

\section{Introduction}

Charge-density studies of high-resolution structures not only enable the deduction of atomic coordinates and displacement parameters, but also provide insight into intermolecular and intramolecular interactions. Characterization of chemical bonds and of charge transfer between molecules and evaluation of the electrostatic potential have been emphasized as outcomes of high-resolution diffraction experiments on biological macromolecules (Dauter et al., 1997; Schmidt \& Lamzin, 2002; Vrielink \& Sampson, 2003; Petrova \& Podjarny, 2004; Lecomte et al., 2005). Such analyses have improved the knowledge and understanding of agents and reaction processes in chemistry and biology. These contributions are especially valuable for the design of drug molecules in pharmacy and medicine. To date, charge-density studies have mainly been performed on small molecules owing to the demanding requirements of the experiment, on the quality of the sample and of the evaluation of the data as listed in Koritsánszky \& Coppens (2001). Recently, several highresolution data sets from more complex structures have been studied (Jelsch et al., 1998, 2000; Housset et al., 2000; Lecomte et al., 2004; Afonine et al., 2007; Dittrich, Koritsánszky et al., 2007; Guillot et al., 2008). However, apart from the study of vitamin $\mathrm{B}_{12}$ (Dittrich, Koritsánszky et al., 2007), the present study on cyclosporine A is to our knowledge the first complete and detailed charge-density study of a medium-sized molecule where these requirements have been met and where no $B$-factor cutoff was applied to exclude atoms from multipole refinement. The structure of cyclosporine A contains 199
Received 14 July 2008

Accepted 2 December 2008 
Table 1

Crystal and structure-refinement data for cyclosporine A.

\begin{tabular}{|c|c|c|}
\hline Temperature (K) & 5 & 90 \\
\hline Empirical formula & \multicolumn{2}{|c|}{$\mathrm{C}_{62} \mathrm{H}_{111} \mathrm{~N}_{11} \mathrm{O}_{12 \cdot \frac{3}{4}} \mathrm{H}_{2} \mathrm{O}$} \\
\hline Formula weight $\left(\mathrm{g} \mathrm{mol}^{-1}\right)$ & \multicolumn{2}{|c|}{1216.3} \\
\hline Cell setting & \multicolumn{2}{|l|}{ Orthorhombic } \\
\hline Space group, $Z$ & \multicolumn{2}{|l|}{$P 2_{1} 2_{1} 2_{1}, 4$} \\
\hline \multicolumn{3}{|l|}{ Unit-cell parameters } \\
\hline$a(\AA)$ & $12.496(2)$ & $12.515(3)$ \\
\hline$b(\AA)$ & $15.645(2)$ & $15.644(3)$ \\
\hline$c(\AA)$ & $35.653(3)$ & $35.728(7)$ \\
\hline$\alpha=\beta=\gamma\left({ }^{\circ}\right)$ & 90.0 & 90.0 \\
\hline Calculated density $\left(\mathrm{g} \mathrm{cm}^{-3}\right)$ & $1.1590(2)$ & $1.1532(5)$ \\
\hline$F(000)$ & 2654 & 2654 \\
\hline Crystal size (mm) & $0.3 \times 0.3 \times 0.6$ & $0.3 \times 0.3 \times 0.6$ \\
\hline Crystal form, colour & \multicolumn{2}{|c|}{ Rectangular, colourless } \\
\hline Wavelength $(\AA)$ & 0.6261 & 0.6261 \\
\hline Absorption coefficient $\mu\left(\mathrm{mm}^{-1}\right)$ & 0.081 & 0.081 \\
\hline Absorption correction & None & \\
\hline Max. $\theta\left(^{\circ}\right)$ & 34.75 & 31.45 \\
\hline$(\sin \theta / \lambda)_{\max }\left(\AA^{-1}\right)$ & 0.91 & 0.83 \\
\hline No. of measured reflections & 246948 & 469924 \\
\hline No. of independent reflections & 40848 & 29793 \\
\hline No. of observed reflections & 40377 & 29582 \\
\hline Criterion for observed reflections & $I>3 \sigma(I)$ & \\
\hline Overall completeness (\%) & $92.3(82.3)$ & $87.5(71.8)$ \\
\hline Redundancy & 6.1 & 15.8 \\
\hline Weighting scheme & $w=\sigma^{-2}\left(F_{\mathrm{obs}}^{2}\right)$ & \\
\hline$R_{\mathrm{mrgd}}\left(F^{2}\right)$ & $0.008(0.006)$ & $0.011(0.021)$ \\
\hline Refinement on & $F^{2}$ & $F^{2}$ \\
\hline$R(F)_{\text {multipole }}$ & 0.0246 & 0.0193 \\
\hline$w R(F)_{\text {multipole }}$ & 0.0291 & 0.0238 \\
\hline$R\left(F^{2}\right)_{\text {multipole }}$ & 0.0410 & 0.0318 \\
\hline$w R\left(F^{2}\right)_{\text {multipole }}$ & 0.0571 & 0.0467 \\
\hline$S_{\text {multipole }}(\mathrm{GoF})$ & 3.70 & 4.71 \\
\hline$\Delta \rho_{\max }\left(\mathrm{e} \AA^{-3}\right)$ & 0.257 & 0.204 \\
\hline$\Delta \rho_{\min }\left(\mathrm{e} \AA^{-3}\right)$ & -0.252 & -0.146 \\
\hline$\Delta \rho_{\text {r.m.s. }}\left(\mathrm{e}^{-3}\right)$ & 0.036 & 0.024 \\
\hline$N_{\text {ref }} / N_{\text {var }}$ & 31.57 & 23.13 \\
\hline
\end{tabular}

Table 2

Data-collection parameters for cyclosporine A measured at $5 \mathrm{~K}$.

\begin{tabular}{llllll}
\hline Run & 1 & 2 & 3 & 4 & 5 \\
\hline Energy (keV) & 19.8 & 19.8 & 19.8 & 19.8 & 19.8 \\
$\Delta \varphi\left(^{\circ}\right)$ & 2 & 2 & 2 & 2 & 2 \\
Exposure time (s) & 2 & 2 & 2 & 2 & 4 \\
No. of frames & 180 & 180 & 180 & 180 & 180 \\
Detector distance (mm) & 80 & 120 & 140 & 150 & 150 \\
Detector tilt in $2 \theta\left({ }^{\circ}\right)$ & 0 & 0 & 0 & 38 & 38 \\
Resolution limits $(\AA)$ & $50-0.9$ & $50-1.25$ & $50-1.5$ & $50-0.45$ & $50-0.45$ \\
\hline
\end{tabular}

atoms (86 non-H atoms) in the asymmetric unit. We believe that a study on larger molecules of biological interest such as cyclosporine A is mandatory in order to clarify the feasibility of the methodology involved, especially since high-resolution studies are now gaining increased attention. In medicine, cyclosporine $\mathrm{A}$ is an immunosuppressant drug that is widely used in post-allogeneic organ transplants. The molecule binds to the cytosolic protein cyclophiline of immunocompetent lymphocytes, especially T-lymphocytes (Lüllmann et al., 2003; Hartmann, 2003). The first X-ray diffraction and spectroscopic experiments on cyclosporine A were performed in 1976 (Petcher et al., 1976; Rüegger et al., 1976). Many X-ray and neutron diffraction as well as NMR experiments have since followed. Among the various studies on cyclosporine A in the literature, we wish to emphasize the following seminal papers: Petcher et al. (1976), Loosli et al. (1985), Knott et al. (1990), Pohl et al. (1995) and Husák et al. (1996, 2000). Studies on the complex of cyclosporine A and cyclophiline include those of Weber et al. (1991), Spitzfaden et al. (1992), Mikol et al. (1993, 1994), Thériault et al. (1993), Pflügl et al. (1993), Mikol \& Duc (1994), Wenger et al. (1994), Knott et al. (1995) and Kallen et al. (2005).

\section{Experimental}

Cyclosporine A was crystallized in its orthorhombic crystal form. The crystal dimensions were $0.3 \times 0.3 \times 0.6 \mathrm{~mm}$. Crystallization was achieved following the protocol described by Novartis (1989). Commercially available cyclosporine A (Fluka Germany; CAS No. 59865-13-3) was dissolved in polyethylene glycol (PEG 400) at a temperature of $323 \mathrm{~K}$. Crystals were obtained by slow cooling of the solution to $293 \mathrm{~K}$.

Several cyclosporine A intensity data sets were collected at beamline X10SA at the Swiss Light Source (SLS, Paul Scherrer Institute, Villigen, Switzerland). Details of two experiments are summarized in Table 1. These experiments were performed at two temperatures of $T=5 \mathrm{~K}$ and $T=90 \mathrm{~K}$ using an open-flow helijet cryostat (CRYO Industries of America, Inc.) and an open-flow nitrogen cryostat (Oxford Instruments $\mathrm{GmbH}$ ), respectively. The resolution achieved in the experiment performed at $5 \mathrm{~K}$ exceeds that of the $90 \mathrm{~K}$ experiment, which is reflected in the larger number of unique reflections. Diffraction images were recorded using a MAR mosaic area detector $(225 \times 225 \mathrm{~mm})$ at a wavelength of $0.6261 \AA$. The beam size was adjusted to $50 \times 150 \mu \mathrm{m}$ (horizontal $\times$ vertical, FWHM). $50 \mu \mathrm{m}$ is the smallest horizontal beamsize possible on beamline X10SA. In order to avoid possible radiation damage owing to the extremely high flux densities, the beam was vertically defocused to $150 \mu \mathrm{m}$. In this scheme the beam is smaller than the crystal for all rotations, as opposed to the often-applied scheme in which the crystal is smaller than the beam. As can be seen in Table 1, the applied scheme resulted in quite decent merging $R$ values for the intensity data. The small horizontal beam size further allowed translation of the crystal along the rotation axis between different data-collection runs to further reduce possible radiation damage. No indications of radiation damage were found during individual runs.

The data-collection strategy consisted of $\varphi$ rotations of $180^{\circ}$ and $360^{\circ}$, respectively. Various sample-to-detector distances in combination with different exposure times and beam attenuations were applied in order to cope with saturated or overloaded detector pixels. In order to cover a higher angular range the detector was rotated by $38^{\circ}$ in $2 \theta$ by a combined movement of the detector tilt and detector distance for collection of the highest resolution intensity data sets. In this way, complete and redundant high-resolution data were obtained. For further details, see Tables 2 and 3. 
Table 3

Data-collection parameters for cyclosporine A measured at $90 \mathrm{~K}$.

\begin{tabular}{lllllll}
\hline Run & 1 & 2 & 3 & 4 & 5 & 6 \\
\hline Energy (keV) & 19.8 & 19.8 & 19.8 & 19.8 & 19.8 & 19.8 \\
$\Delta \varphi\left(^{\circ}\right)$ & 2 & 2 & 2 & 2 & 1 & 1 \\
Exposure time (s) & 2 & 1 & 2 & 2 & 1 & 1 \\
No. of frames & 180 & 180 & 180 & 180 & 360 & 180 \\
Detector distance (mm) & 80 & 120 & 140 & 150 & 150 & 150 \\
Detector tilt in 2 $\theta\left({ }^{\circ}\right)$ & 0 & 0 & 0 & 38 & 38 & 38 \\
Resolution limits $(\AA)$ & $50-0.7$ & $50-1.0$ & $50-1.2$ & $50-0.51$ & $50-0.51$ & $50-0.51$ \\
\hline
\end{tabular}

Data were processed using the program $X D S$ (version 2006; Kabsch, 1993). For the low-dose exposures (runs 1-3), highresolution cutoffs were applied as listed in Tables 2 and 3.

Presently available CCD detectors are optimized for photon energies up to $12 \mathrm{keV}$, where photons are almost completely absorbed by the fluorescence phosphor independent of the incident angle. For higher photon energies the absorption becomes incomplete and a smaller portion of the diffracted photons is absorbed within the phosphor, leading to systematic errors in the measured intensities. The need for an adequate correction has previously been mentioned by Watkin (1994) and possible methods have been suggested (Hammersley et al., 1994; Moy et al., 1996; Zaleski et al., 1998; Wu et al., 2002). In order to take this effect into account, a new correction procedure was applied (Johnas et al., 2006) that has already briefly been described in Meents et al. (2008). It is based on comparison of the intensity data of a standard crystal. This crystal was measured with the area detector used in the experiments and also with a point detector at the same wavelength. Owing to the measurement geometry, the intensities determined with the point detector were not affected by a systematic error arising from the oblique incidence of incoming X-rays and were therefore used as a reference data set. The dependence of the ratio of the reflection intensities on the angle of incidence on the CCD-detector surface was parametrized using an exponential function. This fit function was subsequently applied to the cyclosporine A intensity data sets. The detailed procedure will be published elsewhere.

\section{Electron-density modelling}

Experimental data sets were modelled using the multipole formalism of Hansen \& Coppens (1978) as implemented in the program package $X D$ (Volkov et al., 2006), which was used throughout this study. This formalism describes the total electron density as a sum of a spherical core, a spherical valence and an aspherical deformation density,

$$
\begin{aligned}
\rho_{\text {atom }}(\mathbf{r})=\rho_{\text {core }}(\mathbf{r}) & +P_{\text {val }} \kappa^{3} \rho_{\text {val }}(\kappa \mathbf{r}) \\
& +\sum_{l=0}^{l_{\max }} \kappa^{\prime 3} R_{l}\left(\kappa^{\prime} \mathbf{r}\right) \sum_{m=0}^{l} P_{l m \pm} d_{l m \pm}(\theta, \varphi),
\end{aligned}
$$

where $l$ is the order of the multipole expansion, $P_{\mathrm{val}}, P_{l m}, \kappa$ and $\kappa^{\prime}$ are refinable multipole parameters, $R_{l}$ are densitynormalized Slater-type radial functions and $d_{l m}$ are orientation-dependent spherical harmonic functions. A further program (MOPRO; Guillot et al., 2001) has been developed for the multipole refinement of high-resolution intensity data sets from small proteins. However, it is beyond the scope of this work to present a comparison of different refinement programs.

The thermal motion of atoms is usually described by a harmonic potential. Although this approximation is valid in most cases, it is only a simplification. In order to describe more complicated probability distributions, the potential must be assumed to be anharmonic. Atomic displacement parameters then become complex quantities. If the deviations from harmonic behaviour are small, the anharmonic thermal motion can be described using the Gram-Charlier expansion (Johnson \& Levy, 1974), which is an expansion of the normal distribution including its partial derivatives (Kendal \& Stuart, 1958). In the structure of cyclosporine A some atoms were found to require modelling using an anharmonic potential. These atoms were modelled using the Gram-Charlier expansion up to the third order. If the obtained resolution of the intensity data of a structure of interest does not fulfil the requirements of a charge-density study and if the data-toparameter ratio is not sufficient for a free refinement of charge-density parameters, predicted multipole population parameters from databases can be used to approximate the electron-density distribution. The latest developments of such databases have recently been reported (Zarychta et al., 2007; Dittrich et al., 2006; Volkov et al., 2007) and an overview of their characteristics is given in Table 4. Several other publications investigating small-molecular structures have supported the transferability of the predicted populations in these databases. We will focus on two databases in this work: (i) the invariom database (Dittrich et al., 2004) and (ii) the University at Buffalo Databank (UBDB; Volkov et al., 2004, 2007; Dominiak et al., 2007); the experimental library of multipole moments (ELMAM; Zarychta et al., 2007) will not form part of this investigation. In the following, their suitability for application to larger molecules will be investigated using the data from cyclosporine A.

\subsection{Multipole refinement}

Starting atomic parameters were obtained by sphericalatom refinement using the program SHELXL97 (Sheldrick, 2008) and subsequent spherical-atom refinements using the program $X D$. The quantity $\sum_{\mathbf{h}} w\left(F_{\text {obs }}^{2}-k F_{\text {cal }}^{2}\right)^{2}$ was minimized in all refinements (multipole, invariom, UBDB) using the statistical weighting scheme $w=\sigma^{-2}\left(F_{\text {obs }}^{2}\right)$. Non-H atoms were refined using anisotropic thermal displacement parameters and $\mathrm{H}$ atoms were refined using isotropic thermal displacement parameters. Hydrogen positions were obtained by fixing $X-\mathrm{H}$ distances to values from neutron diffraction experiments (Allen et al., 2004). Three refinement models were compared. In model 1 multipole parameters were freely refined. In models $\mathbf{2}$ and $\mathbf{3}$ multipole parameters were transferred from the invariom database (model 2) and the UBDB (model 3) and kept fixed. In order to reduce the number of parameters in the free refinement $\mathbf{1}$, chemical constraints were applied to atoms of equal element type with identical chemical 
Table 4

Comparison of the three databases that are currently available.

\begin{tabular}{|c|c|c|c|}
\hline & Invariom database & UBDB & ELMAM \\
\hline Transferability 'cutoff' & Empirical rules & Statistics & Empirical findings/statistics \\
\hline Database construction & $\begin{array}{l}\text { Geometry optimizations of model } \\
\text { compounds }\end{array}$ & $\begin{array}{l}\text { Single-point energy calculations of } \\
\text { structures from the CSD }\end{array}$ & $\begin{array}{l}\text { Multipole refinements of high-resolution } \\
\text { X-ray data }\end{array}$ \\
\hline Method used & B3LYP, basis D95++(3df,3pd) & B3LYP, basis $6-31 \mathrm{G}^{*}$ & $\mathrm{X}$-ray diffraction \\
\hline Main aim & $\begin{array}{l}\text { Structure refinement and property } \\
\text { calculation }\end{array}$ & $\begin{array}{l}\text { Structure refinement and property } \\
\text { calculation }\end{array}$ & $\begin{array}{l}\text { Starting values for protein multipole } \\
\text { refinement }\end{array}$ \\
\hline Known strengths/weaknesses & $\begin{array}{l}\text { Does not include effects of hydrogen } \\
\text { bonding }\end{array}$ & $\begin{array}{l}\text { Does not include effects of hydrogen } \\
\text { bonding }\end{array}$ & $\begin{array}{l}\text { Includes average effect of hydrogen } \\
\text { bonding }\end{array}$ \\
\hline & $\begin{array}{l}\text { Easily extendable to new chemical } \\
\text { environments }\end{array}$ & $\begin{array}{l}\text { Easily extendable to new chemical } \\
\text { environments }\end{array}$ & $\begin{array}{l}\text { Experiment required for extension of } \\
\text { database }\end{array}$ \\
\hline Organization & Invariom notation & $\begin{array}{l}\text { No direct link between scattering } \\
\text { factor and its name }\end{array}$ & IUPAC notation for protein atoms \\
\hline
\end{tabular}

environment. A further reduction was achieved by the application of local atomic site symmetries. The following atoms were selected according to the rules given by Kurki-Suonio (1977) and were checked against residual densities and convergence: atoms of $C$-methyl and $N$-methyl groups, $\mathrm{C}=\mathrm{O}$ and $\mathrm{C}=\mathrm{C}$ bonds, primary and secondary substructures of $\mathrm{Csp} p^{3}-\mathrm{H}$ bonds, bonds to $\mathrm{H}$ atoms and water molecules (for more details, see Supplementary Table $1^{\mathbf{1}}$ ). In this multipole refinement, no special refinement protocol (as, for example, given in Guillot et al., 2008) was required and the physically most reasonable multipole parameters were obtained using this highly constrained model with all reflections and no $B$-factor cutoff for non- $\mathrm{H}$ atoms. The refinement parameters for non-H atoms included anisotropic displacement parameters and multipole population parameters up to the hexadecapolar level. $\mathrm{H}$ atoms were refined isotropically and up to the quadrupolar level including only bond-directed multipole parameters. The expansion-contraction coefficients $\kappa$ and $\kappa^{\prime}$ were fixed to standard values of 1.0 for non-H atoms and 1.2 for $\mathrm{H}$ atoms, since their free refinement was not successful.

A total of six atom sites in the molecule were found to be disordered in the crystal: three non-H atoms [C(99), C(110) and $\mathrm{O}(112)]$ and three $\mathrm{H}$ atoms [H(79A), $\mathrm{H}(79 \mathrm{~B})$ and $\mathrm{H}(79 \mathrm{C})]$. The disordered $\mathrm{H}$ atoms were refined using the conventional split method, which takes two possible positions with different occupations into account with an occupancy sum of 1.0. The three disordered $\mathrm{H}$ atoms are bonded to $\mathrm{N}(7)$. The thermal motion of the disordered non-H atoms C(99), $\mathrm{C}(110)$ and $\mathrm{O}(112)$ was considered to be anharmonic and was described by the Gram-Charlier expansion up to the third order, assuming local site symmetry 1 . An alternative refinement with split positions of these atoms gave comparable results in terms of the achievable residual densities and $R$ values. Owing to the small distance between the split positions ( $\leq 0.4 \AA)$, the evaluation was continued with anharmonic displacement parameters. Charge transfer was allowed between cyclosporine $\mathrm{A}$ and the water molecule, with the complete charge of the unit cell being kept constant. One scale factor was refined for the whole data set. This refinement

\footnotetext{
${ }^{1}$ Supplementary material has been deposited in the IUCr electronic archive (Reference: DZ5137). Services for accessing this material are described at the back of the journal.
}

strategy was applied to both experimentally obtained data sets ( 5 and $90 \mathrm{~K}$ ). A total of 1279 parameters were refined. The refined $R$ value for the data set measured at $5 \mathrm{~K}$ is about $0.5 \%$ higher than that of the data set measured at $90 \mathrm{~K}$. Since the merged $R$ values of both data sets are comparable, the exact reason for this behaviour is not obvious. One reason could be the statistical noise introduced by the higher resolution $5 \mathrm{~K}$ data set with a larger fraction of weak high-resolution reflections. The reflection-to-parameter ratio was almost 32 for the data set measured at $5 \mathrm{~K}$ and was 23 for the data set measured at $90 \mathrm{~K}$ (see Table 1).

\subsection{Pseudoatom modelling: invariom database and University at Buffalo Databank}

The same starting geometry was again obtained from SHELXL97. Using the programs InvariomTool (Hübschle et al., 2007) and LSDB (Volkov et al., 2004, 2007; Dominiak et al., 2007), multipole population parameters, $\kappa$ sets, local symmetry restraints and chemical constraints were automatically transferred from the invariom database and the UBDB, respectively. Only positional coordinates and displacement parameters as well as one scale factor were refined (refinements 2 and 3). The multipole populations from both databases were kept constant during the two refinements. Analogous to the multipole refinement, bond distances involving $\mathrm{H}$ atoms were elongated to standard values from neutron diffraction experiments (Allen et al., 2004). As before, the atomic displacement parameters of disordered non-H atoms were described by a Gram-Charlier expansion. For each type of pseudoatom modelling, the same level of the multipole expansion was considered as in the free refinement 1. H-atom multipole populations were also assumed to contribute in bond direction only.

In comparison to the sum of neutral atoms in one cyclosporine A molecule including the partially occupied water molecule, the sum of the monopole populations in the two databanks differ slightly. This leads to a difference of less than $0.80 \%$ of the number of valence electrons in the invariom database and to a difference of $0.45 \%$ in the UBDB. Since the databanks are based on different selection rules to identify an atom in its chemical environment, different local symmetries were assumed to some extent. Overall, our impression was 
that higher local atomic site symmetries are applied in the invariom database than in the UBDB (see Supplementary Tables 2 and 3). Both choices of symmetry were applicable to the molecular structure of cyclosporine A.

\section{Results and discussion}

The molecular structure in the crystal and the numbering scheme of cyclosporine A are depicted in Figs. 1( $a)$ and 1(b). Full topological analyses of the different density models 1-3 were carried out using the method of Bader (1990). In the following, we concentrate on the results for data measured at 5 and $90 \mathrm{~K}$ in order to prove the feasibility of charge-density analysis of medium-sized molecules. We verify these results by comparing them with previous studies on small molecules from the literature. Finally, the predicted multipole populations from the two databases were verified. Their performance in modelling the charge density of cyclosporine $\mathrm{A}$ was compared with the free multipole refinement $\mathbf{1}$.

\subsection{Multipole refinement and topological analyses of data sets measured at $T=5 \mathrm{~K}$ and $T=90 \mathrm{~K}$}

Refinement of the multipole model against the measured squared structure factors at temperatures of 5 and $90 \mathrm{~K}$ yielded excellent figures of merit (see Table 1). Residual densities were checked and showed no significant features,

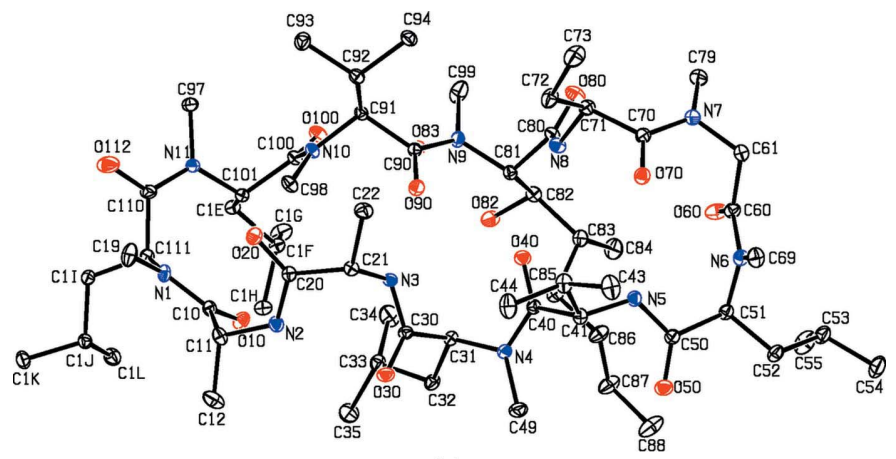

(a)

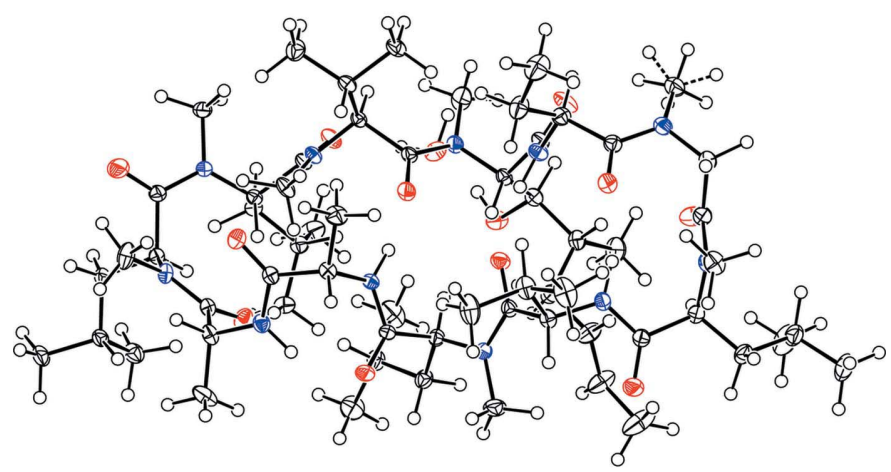

(b)

Figure 1

ORTEP representation (Burnett \& Johnson, 1996) of (a) cyclosporine A measured at $T=5 \mathrm{~K}$ with the atomic numbering scheme omitting $\mathrm{H}$ atoms for clarity and (b) cyclosporine A measured at $T=90 \mathrm{~K}$ including $\mathrm{H}$ atoms, which are numbered after the atoms to which they are bonded to plus a capital letter to distinguish them. Thermal displacement ellipsoids are shown at a $50 \%$ probability. which was supported by the low values of the minimal and maximal residual electron densities, especially when taking into account the size of the structure and the three disordered or anharmonic non-H atoms. Thus, adequate modelling of $F^{2}$ was affirmed. Deformation electron densities from the refined multipole parameters based on (1) displayed the expected concentrations on chemical bonds and at lone pairs. Furthermore, residual density maps, i.e. dynamic deformation electron-density maps, although thermally smeared, also showed the presence of these features. Both models fulfilled the rigid-bond test of Hirshfeld (the difference of the meansquare displacement amplitudes DMSDA was $\leq 0.001 \AA^{2}$; Hirshfeld, 1976). Only small variations are observed at the sites of the disordered oxygen $\mathrm{O}(112)$ and carbon $\mathrm{C}(110)$. However, this can be ascribed to the anharmonic oscillations of these atoms, to possible parameter correlations or to inadequacies of the anharmonic model. Even these values are still significantly smaller than those for nonrigid systems (Rosenfield et al., 1978; Dunitz et al., 1988). Thus, adequate separation of atomic displacement parameters and deformation electron density of the valence shell can be assumed to have been successful.

Owing to the different temperatures of the experiments the atomic displacement factors of the two data sets differ. On average, the atomic displacement factors deduced from the data set measured at $T=90 \mathrm{~K}$ are about $50 \%$ larger than those from the data set measured at $T=5 \mathrm{~K}$ (see Fig. 1). On comparing the refined population parameters of both multipole refinements, no significant differences were observed.

A complete topological analysis of the modelled static charge density of each data set was carried out. All characteristics deduced from this analysis agree with and confirm previous studies on small molecules. Thus, we would like to emphasize that studies of this type are indeed feasible for medium-sized molecules. This allows an optimistic outlook for future studies on high-resolution protein structures. Furthermore, no significant changes to topological descriptors such as bond critical points were found for the two data sets (measured at $T=5$ and $90 \mathrm{~K}$; see Supplementary Table 3). Hence, the results of these analyses on cyclosporine A confirm their correctness and reliability. Owing to the higher resolution of the structural model obtained from the data set measured at $T=5 \mathrm{~K}$ and the smaller atomic displacement parameters in this model, and owing to the almost identical results that were obtained, we will only discuss the results of the data set measured at $T=5 \mathrm{~K}$ in more detail.

In order to compare the experimental results with studies on small molecules from the literature, we calculated the mean average values of the charge density $\overline{\rho(\mathbf{r})_{\mathrm{CP}}}$ and the Laplacian $\overline{\nabla^{2} \rho(\mathbf{r})_{\mathrm{CP}}}$ on bond critical points for common bonds in the main chain and the side chains of the various amino acids as well as their standard deviations ${ }^{2}$ (Table 5). A quantitative comparison with published experimental studies on 16 different amino acids as well as theoretical calculations on 20

\footnotetext{
${ }^{2}$ Each standard deviation of the mean average $\bar{x}=(1 / n) \sum_{i=1}^{n} x_{i}$ is given by the formula $\sigma^{2}(\bar{x})=1 /[n(n-1)] \sum_{i=1}^{n}\left(x_{i}-\bar{x}\right)^{2}$.
} 
Table 5

Mean average of the charge density $\overline{\rho(\mathbf{r})_{C P}}$ and its Laplacian $\overline{\nabla^{2} \rho(\mathbf{r})_{C P}}$ at the bond critical point, together with the standard deviation of the mean average $\sigma\left[\overline{\rho(\mathbf{r})_{C P}}\right]$ and $\sigma\left[\overline{\nabla^{2} \rho(\mathbf{r})_{C P}}\right]$, respectively.

The number of bonds taken into account is also given. The first line (Exp.) gives the results from experimental multipole refinement of cyclosporine A measured at $T=5 \mathrm{~K}$. The second line (Lit. exp.) gives the mean average and standard deviations of published experimental studies on 16 amino acids (Mebs et al., 2006). The third line (Lit. Theo.) gives the mean average and standard deviations of theoretical calculations on 20 amino acids (Matta \& Bader, 2003). Disordered atoms were not taken into account for the mean averages.

\begin{tabular}{lllllll}
\hline $\begin{array}{l}\text { Bond } \\
\text { type }\end{array}$ & $\begin{array}{l}\overline{\rho(\mathbf{r})_{\mathrm{CP}}} \\
\left(\mathrm{e} \AA^{-3}\right)\end{array}$ & $\begin{array}{l}\sigma\left[\overline{\rho(\mathbf{r})_{\mathrm{CP}}}\right] \\
\left(\mathrm{e}^{-3}\right)\end{array}$ & $\begin{array}{l}\overline{\nabla^{2} \rho(\mathbf{r})_{\mathrm{CP}}} \\
\left(\mathrm{e}^{-5}\right)\end{array}$ & $\begin{array}{l}\sigma\left[\overline{\nabla^{2} \rho(\mathbf{r})_{\mathrm{CP}}}\right] \\
\left(\mathrm{e}^{-5}\right)\end{array}$ & $\begin{array}{l}\text { No. of } \\
\text { bonds }\end{array}$ & Source \\
\hline $\mathrm{C}=\mathrm{O}$ & 2.77 & 0.01 & -31.7 & 0.6 & 10 & Exp. \\
& 2.86 & 0.03 & -35.6 & 1.2 & 16 & $\begin{array}{l}\text { Lit. Exp. } \\
\end{array}$ \\
\hline $\mathrm{C}^{\alpha}-\mathrm{N}$ & 1.94 & 0.01 & 2.5 & 0.1 & 24 & Lit. Theo. \\
& 1.69 & 0.02 & -10.5 & 0.8 & 11 & Exp. \\
& 1.90 & 0.01 & -10.5 & 0.7 & 17 & Lit. Exp. \\
\hline $\mathrm{C}^{\alpha}-\mathrm{CO}$ & 1.70 & 0.02 & -11.6 & 0.6 & 10 & Exp. \\
& 1.74 & 0.02 & -12.6 & 0.7 & 17 & Lit. Exp. \\
& 1.82 & 0.01 & -19.2 & 0.1 & 24 & Lit. Theo. \\
\hline $\mathrm{C}^{\alpha}-\mathrm{C}^{\beta}$ & 1.65 & 0.02 & -10.8 & 0.3 & 10 & Exp. \\
& 1.68 & 0.02 & -11.2 & 0.5 & 16 & Lit. Exp. \\
& 1.71 & 0.01 & -16.3 & 0.1 & 24 & Lit. Theo. \\
\hline
\end{tabular}

different amino acids (Matta \& Bader, 2003) has been summarized by Mebs et al. (2006). For the theoretical calculations the same method was used [restricted Hartree-Fock calculations with the program Gaussian 94 (Frisch et al., 1995) and the basis set 6-311++G** (Matta \& Bader, 2003)]. Within the main chains of the amino acids in cyclosporine A, four of the five different bond types of the main chains of amino acids occur: $\mathrm{C}=\mathrm{O}, \mathrm{C}=\mathrm{OH}, \mathrm{C}^{\alpha}-\mathrm{N}, \mathrm{C}^{\alpha}-\mathrm{C}^{\prime}(=\mathrm{CCO})$ and $\mathrm{C}^{\alpha}-\mathrm{C}^{\beta}$ (= CCR). Differences in charge densities at bond critical points can arise from a number of factors: different experimental parameters such as, for example, temperature and crystal quality, different refinement strategies or multipole models and also physical effects such as different conformation, crystal packing and hydrogen bonding. The standard deviations of the mean averages of the charge density $\overline{\rho(\mathbf{r})_{\mathrm{CP}}}$ of

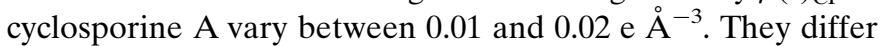
from the values from earlier experimental studies within one to three standard deviations and from values from theoretical calculations within three to nine standard deviations. Thus, good agreement is obtained. Similar behaviour is observed for the averaged Laplacians. Their standard deviations vary between 0.2 and 0.8 e $\AA^{-5}$. When compared with the mean averages of experimental values from Mebs et al. (2006) they are also in good agreement. More pronounced differences are seen with respect to the theoretical calculations of Matta and Bader, which is well known from the literature (Volkov et al., 2000). Owing to inflexibility of the radial functions in the multipole model, values of the Laplacian in particular from experiment and theory can be quite different.

Other bond types within cyclosporine A that were not covered by previous theoretical and experimental studies were also averaged (Table 6) and also showed very good agreement of the electron density and its Laplacian at the bond critical
Table 6

Mean averages of the charge density $\overline{\rho(\mathbf{r})_{\mathrm{CP}}}$ and its Laplacian $\overline{\nabla^{2} \rho(\mathbf{r})_{\mathrm{CP}}}$ at the bond critical point and their standard deviations $\sigma\left[\overline{\rho(\mathbf{r})_{\mathrm{CP}}}\right]$ and $\sigma\left[\overline{\nabla^{2} \rho(\mathbf{r})_{\mathrm{CP}}}\right]$.

The number of bonds taken into account is also given. The $\mathrm{N}-\mathrm{C}$ bond represents the peptide bond.

\begin{tabular}{llllll}
\hline Bond type & $\begin{array}{l}\overline{\rho(\mathbf{r})_{\mathrm{CP}}} \\
\left(\mathrm{e} \AA^{-3}\right)\end{array}$ & $\begin{array}{l}\sigma\left[\overline{\rho(\mathbf{r})_{\mathrm{CP}}}\right] \\
\left(\mathrm{e} \AA^{-3}\right)\end{array}$ & $\begin{array}{l}\overline{\nabla^{2} \rho(\mathbf{r})_{\mathrm{CP}}} \\
\left(\mathrm{e}^{-5}\right)\end{array}$ & $\begin{array}{l}\sigma\left[\overline{\nabla^{2} \rho(\mathbf{r})_{\mathrm{CP}}}\right] \\
\left(\mathrm{e}^{-5}\right)\end{array}$ & No. of bonds \\
\hline $\mathrm{N}-\mathrm{C}$ & 2.25 & 0.01 & -22.1 & 0.4 & 10 \\
$\mathrm{~N}-\mathrm{CH}_{3}$ & 1.73 & 0.02 & -13.4 & 0.8 & 5 \\
$\mathrm{C}-\mathrm{OH}$ & 1.66 & - & -5.1 & - & 1 \\
$\mathrm{C}=\mathrm{C}$ & 2.30 & - & -20.6 & - & 1 \\
$\mathrm{C}-\mathrm{C}$ & 1.66 & 0.01 & -11.1 & 0.2 & 22 \\
\hline
\end{tabular}

point. These also compare well with studies in the literature (Flaig et al., 2002; Rödel et al., 2006). Different covalent bond types were compared; the $\mathrm{C}=\mathrm{O}$ bond is the strongest, with an average value of $\rho(\mathbf{r})_{\mathrm{CP}}=2.77$ (2) e $\AA^{-3}$. The electron density at the bond critical point between the disordered atoms

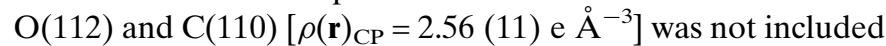
in this average value. Small variations of bond critical point properties in the $\mathrm{C}=\mathrm{O}$ bond can be attributed to hydrogen bonding and also to inadequacies of the multipole model (Volkov et al., 2000). The second strongest bond type appearing is the carbon-carbon double bond. It is considerably stronger than a carbon-carbon single bond, as expected from the literature (Sykes, 1988). Furthermore, peptide bonds are stronger than $\mathrm{C}^{\alpha}-\mathrm{N}$ bonds. This seems to be reasonable from a chemical point of view because of delocalization and the different chemical environments, i.e. the additional polarization of the $\mathrm{C}$ atom of the peptide bond by the $\mathrm{O}$ atom.

After having quantified the topological parameters of cyclosporine $\mathrm{A}$, we can draw the conclusion that the results derived from the two data sets are in good agreement. They also agree with the literature values from studies on small molecules. To summarize, the data are very well suited for a charge-density analysis and enable detailed insight into the atomic and bond topological properties of the molecule in the crystal. Having assessed the data quality, we next compared the predicted multipole populations (models 2 and 3 ) from the databases with the refined multipole parameters (model 1).

\subsection{Application of multipole populations from databases}

In the following, only the data set measured at $T=5 \mathrm{~K}$ was evaluated and discussed for the reasons mentioned earlier. The figures of merit for the refinement of atomic coordinates and displacement parameters using the theoretically predicted multipole population parameters from the two databases (invariom and UBDB) are summarized in Table 7. The two refinements show a comparable performance with respect to the Hirshfeld test (Hirshfeld, 1976). The refined coordinates of the different models generally showed differences that were smaller than one standard deviation. Furthermore, the figures of merit for an IAM refinement and a multipole refinement of the data sets are listed. As expected, the figures of merit for the IAM refinement are worse than those from the three multipole refinements. From the figures of merit one can see 
that both databases perform similarly in fitting the nonspherical electron density. To our knowledge, this is the first study that provides such evidence. Since the database parameters do not include the effects of hydrogen bonding, these figures are not as good as those obtained from the free multipole refinement 1. An alternative explanation is that refinement $\mathbf{1}$ simply includes more parameters. Furthermore, differences in the performance of the databases will be affected by the basis set used [invariom, D95++(3df,3pd); UBDB, 6-31G*], although the projection onto the multipole model does not permit full use of the more extended basis. In any case, database refinements are far better than the IAM refinement and do not increase the number of parameters to be refined. These findings compare well with previous studies (Dittrich, Munshi et al., 2007; Kalinowski et al., 2007; Volkov et al., 2007).

To directly compare the multipole populations, the differences in the static electron density obtained from refinement $\mathbf{1}$ and each database refinement (2 or 3 ) were calculated (Dittrich, Munshi et al., 2007). For this difference density it was essential that the same molecular geometry was chosen in all three cases. The electron density on a grid was calculated with the program $X D P R O P$ and differences were obtained

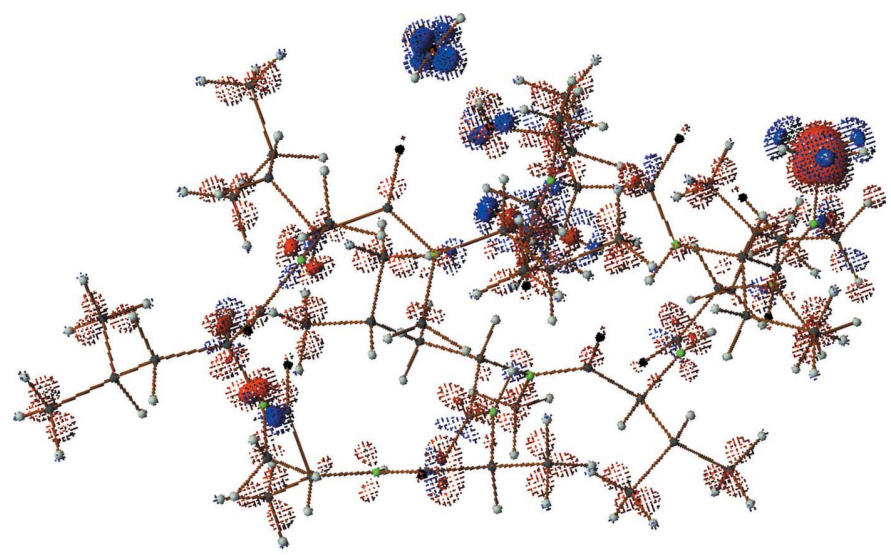

(a)

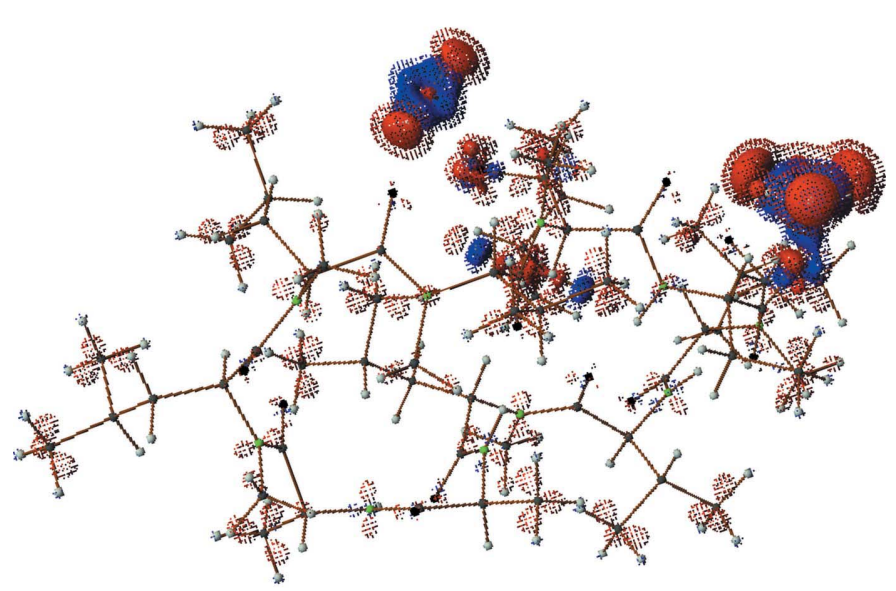

Figure 2

(b)

Calculated differences between multipole and invariom refinement $(a)$ and multipole and UBDB refinement $(b)$. The isosurfaces shown here are

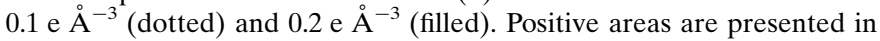
blue and negative areas in red.
Table 7

Figures of merit.

\begin{tabular}{lllll}
\hline Model & IAM & Multipole & Invariom & UBDB \\
\hline No. of observed reflections & 40377 & 40377 & 40377 & 40377 \\
Refinement on & $F^{2}$ & $F^{2}$ & $F^{2}$ & $F^{2}$ \\
Weighting scheme & $w=\sigma^{-2}\left(F_{\text {obs }}^{2}\right)$ & & \\
$R(F)$ & 0.0339 & 0.0246 & 0.0263 & 0.0264 \\
$w R(F)$ & 0.0461 & 0.0291 & 0.0329 & 0.0333 \\
$R\left(F^{2}\right)$ & 0.0555 & 0.0410 & 0.0436 & 0.0441 \\
$w R\left(F^{2}\right)$ & 0.0894 & 0.0571 & 0.0642 & 0.0646 \\
$\mathrm{GoF}$ & 5.77 & 3.70 & 4.14 & 4.17 \\
$\Delta \rho_{\text {max }}\left(\mathrm{e} \AA^{-3}\right)$ & 0.445 & 0.257 & 0.277 & 0.260 \\
$\Delta \rho_{\text {min }}\left(\mathrm{e} \AA^{-3}\right)$ & -0.299 & -0.252 & -0.324 & -0.312 \\
$\Delta \rho_{\text {r.m.s. }}\left(\mathrm{e} \AA^{-3}\right)$ & 0.049 & 0.036 & 0.039 & 0.039 \\
$N_{\text {ref }} / N_{\text {var }}$ & 43.84 & 31.57 & 43.84 & 43.84 \\
\hline
\end{tabular}

using the utility ADDGRID (Volkov et al., 2006). The two figures (see Fig. 2) show similar features and only small deviations are noticeable. These differences are not residual densities since only modelled density was taken into account. In each figure the main differences are observed at the partially occupied water molecule and in an area containing disordered $\mathrm{H}$ atoms in propeller configuration. This indicates inadequate modelling of disordered sites and the partially occupied water molecule by the multipole model used; attempts to further improve this model were unsuccessful. Another critical issue is hydrogen bonding, which is only included in free multipole refinement of the X-ray diffraction data (refinement 1). Further systematic differences can be seen in both figures at the methyl carbon groups. However, the

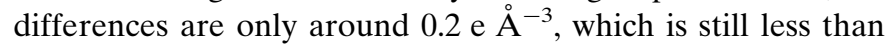
the residual densities of the refinements (compare with Table 7). One can conclude that both database populations are equally suitable for modelling the charge density of such a structure. Considering that the size of the molecule approaches that of protein molecules, the application to even larger structures seems to be justified.

To complement our analysis, we also compared the electron densities and Laplacians at the bond critical points for refinements 1-3. As in the difference density plots, we chose the same geometry to allow a direct comparison between experimental and database results. The mean average of the charge densities and their Laplacians for equal bond types of the main chains and side chains of the amino acids were derived as described above. These are given in Table 8, including their standard deviations. Only small differences occur. The charge density on the bond critical points shows only minor variations, whereas the Laplacians vary a little more, behaviour that has been reported previously (Volkov et $a l ., 2000)$. Thus, the results presented confirm the conclusion that the listed population parameters of the databases are comparable in quality and that either one of them is well suited to modelling the charge density of medium-sized molecules and potentially also of larger molecules.

\section{Conclusion}

In this work, we have described the first detailed and complete charge-density study on the medium-sized molecule cyclo- 
Table 8

Mean average of the charge density $\overline{\rho(\mathbf{r})_{\mathrm{CP}}}$ and its Laplacian $\overline{\nabla^{2} \rho(\mathbf{r})_{\mathrm{CP}}}$ at the bond critical point, together with the standard deviation of the mean average $\sigma\left[\overline{\rho(\mathbf{r})_{\mathrm{CP}}}\right]$ and $\sigma\left[\overline{\nabla^{2} \rho(\mathbf{r})_{\mathrm{CP}}}\right]$, respectively, and the number of bonds taken into account $(n)$.

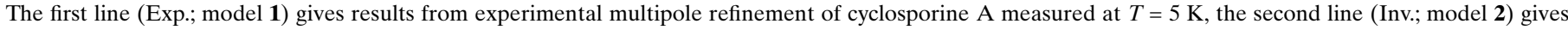

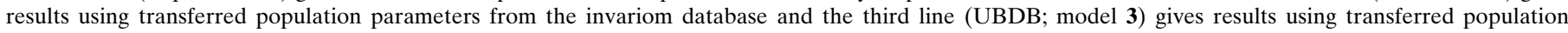
parameters from the UBDB. No disordered atoms are taken into account for the mean averages.

\begin{tabular}{|c|c|c|c|c|c|c|c|c|c|c|}
\hline Bond type & 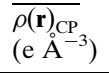 & $\begin{array}{l}\sigma\left[\overline{\rho_{(\mathbf{r})_{\mathrm{CP}}}}\right] \\
\left(\mathrm{e} \AA^{-3}\right)\end{array}$ & $\begin{array}{l}\rho(\mathbf{r})_{\mathrm{CP} \text { min }} \\
\left(\mathrm{e} \AA^{-3}\right)\end{array}$ & $\begin{array}{l}\rho(\mathbf{r})_{\mathrm{CP}, \max } \\
\left(\mathrm{e} \AA^{-3}\right)\end{array}$ & $\begin{array}{l}\overline{\nabla^{2} \rho_{\mathrm{o}}(\mathbf{r})_{\mathrm{CP}}} \\
\left(\mathrm{e} \AA^{-5}\right)\end{array}$ & $\begin{array}{l}\sigma\left[\overline{\nabla^{2} \rho(\mathbf{r})_{\mathrm{CP}}}\right] \\
\left(\mathrm{e} \AA^{-5}\right)\end{array}$ & $\begin{array}{l}\left|\nabla^{2} \rho(\mathbf{r})_{\mathrm{CP}, \min }\right| \\
\left(\mathrm{e} \AA^{-5}\right)\end{array}$ & $\begin{array}{l}\left|\nabla^{2} \rho(\mathbf{r})_{\mathrm{CP}, \max }\right| \\
\left(\mathrm{e} \AA^{-5}\right)\end{array}$ & $n$ & Source \\
\hline \multirow[t]{3}{*}{$\mathrm{C}=\mathrm{O}$} & 2.77 & 0.01 & 2.73 & 2.82 & -31.7 & 0.6 & -29.2 & -33.6 & 10 & Exp. \\
\hline & 2.80 & 0.04 & 2.63 & 2.92 & -32.5 & 0.8 & -29.6 & -35.3 & 10 & Inv. \\
\hline & 2.74 & 0.01 & 2.72 & 2.76 & -24.8 & 0.38 & -23.2 & -26.3 & 10 & UBDB \\
\hline \multirow[t]{3}{*}{$\mathrm{C}^{\alpha}-\mathrm{N}$} & 1.73 & 0.02 & 1.51 & 1.79 & -10.5 & 0.8 & -5.0 & -12.9 & 11 & Exp. \\
\hline & 1.77 & 0.01 & 1.73 & 1.83 & -9.3 & 0.3 & -7.9 & -10.7 & 11 & Inv. \\
\hline & 1.70 & 0.09 & 1.67 & 1.75 & -9.9 & 0.4 & -7.0 & -11.4 & 11 & UBDB \\
\hline \multirow[t]{3}{*}{$\mathrm{C}^{\alpha}-\mathrm{CO}$} & 1.70 & 0.02 & 1.56 & 1.77 & -11.6 & 0.6 & -7.8 & -13.6 & 10 & Exp. \\
\hline & 1.74 & 0.01 & 1.69 & 1.80 & -11.8 & 0.1 & -11.1 & -12.4 & 10 & Inv. \\
\hline & 1.74 & 0.01 & 1.71 & 1.76 & -13.1 & 0.1 & -12.7 & -13.6 & 10 & UBDB \\
\hline \multirow[t]{3}{*}{$\mathrm{C}^{\alpha}-\mathrm{C}^{\beta}$} & 1.65 & 0.02 & 1.58 & 1.74 & -10.8 & 0.3 & -9.5 & -12.95 & 10 & Exp. \\
\hline & 1.67 & 0.01 & 1.64 & 1.74 & -11.4 & 0.2 & -10.8 & -13.2 & 10 & Inv. \\
\hline & 1.65 & 0.01 & 1.62 & 1.69 & -11.5 & 0.1 & -11.0 & -12.5 & 10 & UBDB \\
\hline \multirow[t]{3}{*}{$\mathrm{N}-\mathrm{C}$} & 2.25 & 0.01 & 2.20 & 2.33 & -22.1 & 0.4 & -20.4 & -24.3 & 10 & Exp. \\
\hline & 2.23 & 0.01 & 2.17 & 2.29 & -21.3 & 0.3 & -19.2 & -22.3 & 10 & Inv. \\
\hline & 2.22 & 0.01 & 2.17 & 2.25 & -22.2 & 0.4 & -19.0 & -23.6 & 10 & UBDB \\
\hline \multirow[t]{3}{*}{$\mathrm{N}-\mathrm{CH}_{3}$} & 1.73 & 0.02 & 1.69 & 1.78 & -13.4 & 0.8 & -11.9 & -15.6 & 5 & Exp. \\
\hline & 1.79 & 0.01 & 1.79 & 1.80 & -10.6 & 0.1 & -10.6 & -10.7 & 5 & Inv. \\
\hline & 1.71 & 0.01 & 1.71 & 1.71 & -9.6 & 0.1 & -9.5 & -9.7 & 5 & UBDB \\
\hline \multirow[t]{3}{*}{$\mathrm{C}-\mathrm{OH}$} & 1.66 & - & - & - & -5.1 & - & - & - & 1 & Exp. \\
\hline & 1.80 & - & - & - & -14.5 & - & - & - & 1 & Inv. \\
\hline & 1.75 & - & - & - & -11.9 & - & - & - & 1 & UBDB \\
\hline \multirow[t]{3}{*}{$\mathrm{C}=\mathrm{C}$} & 2.30 & - & - & - & -20.6 & - & - & - & 1 & Exp. \\
\hline & 2.32 & - & - & - & -22.9 & - & - & - & 1 & Inv. \\
\hline & 2.60 & - & - & - & -27.6 & - & - & - & 1 & UBDB \\
\hline \multirow[t]{3}{*}{$\mathrm{C}-\mathrm{C}$} & 1.66 & 0.01 & 1.62 & 1.72 & -11.1 & 0.2 & -8.3 & -12.2 & 22 & Exp. \\
\hline & 1.65 & 0.01 & 1.61 & 1.75 & -10.3 & 0.1 & -9.8 & 11.6 & 22 & Inv. \\
\hline & 1.61 & 0.01 & 1.58 & 1.71 & -10.7 & 0.1 & -10.3 & -11.9 & 22 & UBDB \\
\hline
\end{tabular}

sporine A. Two data sets measured at different temperatures were evaluated. Comparable physical properties were deduced for the two data sets, so we concentrated on the $5 \mathrm{~K}$ data, which were measured to higher resolution and for which the ADPs were smaller. Results from a topological analysis of the experimental electron density were in very good agreement with studies on small molecules from the literature and confirmed chemical expectations.

Particularly for more complex structures, it is very difficult or impossible to obtain high-resolution data. Considerable interest in charge-density results of these structures nevertheless exists. Although a conventional spherical-atom structure refinement is already an achievement in these cases, database approaches that allow an approximate electron density of high quality to be taken into account are now available. The results of such refinements promise to be very interesting. The reliability of two existing databases (the invariom database and the University at Buffalo Databank) has been verified here. The data set measured at $T=5 \mathrm{~K}$ proved to be ideally suited for evaluation of the quality of the database population parameters. It was confirmed that when the geometry is known the transfer of these database parameters enables charge-density results of comparable quality to a free multipole refinement to be obtained, even for a medium-sized molecule such as cyclosporine A. However, as expected, slightly better results were obtained by free refinement of multipole parameters.

Atoms that exhibit harmonic oscillations in the crystal are very well modelled by the multipole model in combination with anisotropic temperature factors. This holds for refined parameters as well as for database models with transfered multipole populations. For atoms that oscillate anharmonically, a phenomenon that occurs more often in larger structures, the charge density can also be modelled satisfactorily by higher-order temperature factors. However, the multipole parameters of these atoms show differences in comparison to non-disordered atoms. As these multipole parameters can correlate with the Gram-Charlier parameters, a careful deconvolution of thermal motion and electron density is especially crucial in these cases. Therefore, refinement of these atoms seems to be more reliable using database population parameters when they are combined with a Gram-Charlier expansion and the results of topological analyses should be evaluated critically for these atoms.

The authors would like to thank Dr Sascha Gutmann for his help and support at beamline X10SA (SLS) and Dr F. P. A. 
Fabbiani for comments on the manuscript. This work was supported by Deutsche Forschungsgemeinschaft DFG grant 921/3-1 and the Australian Synchrotron Research Program, which is funded by the Commonwealth of Australia under the Major National Research Facilities Program.

\section{References}

Allen, F. H., Watson, D. G., Brammer, L., Orpen, A. G. \& Taylor, R. (2004). International Tables for Crystallography, Vol. C, edited by E. Prince, pp. 790-811. Dordrecht: Kluwer Academic Publishers.

Afonine, P. V., Grosse-Kunstleve, R. W., Adams, P. D., Lunin, V. Y. \& Urzhumtsev, A. (2007). Acta Cryst. D63, 1194-1197.

Bader, R. F. W. (1990). Atoms in Molecules: A Quantum Theory. Oxford: Clarendon Press.

Burnett, M. N. \& Johnson, C. K. (1996). ORTEP-III. Oak Ridge National Laboratory Report ORNL-6895. Oak Ridge, Tennessee, USA.

Dauter, Z., Lamzin, V. S. \& Wilson, K. S. (1997). Curr. Opin. Struct. Biol. 7, 681-688.

Dittrich, B., Hübschle, C. B., Luger, P. \& Spackman, M. A. (2006). Acta Cryst. D62, 1325-1335.

Dittrich, B., Koritsánszky, T. \& Luger, P. (2004). Angew. Chem. Int. Ed. 43, 2718-2721.

Dittrich, B., Koritsánszky, T., Volkov, A., Mebs, S. \& Luger, P. (2007). Angew. Chem. Int. Ed. 46, 2935-2938.

Dittrich, B., Munshi, P. \& Spackman, M. A. (2007). Acta Cryst. B63, 505-509.

Dominiak, P. M., Volkov, A., Li, X., Messerschmidt, M. \& Coppens, P. (2007). J. Chem. Theor. Comput. 3, 232-247.

Dunitz, J. D., Schomaker, V. \& Trueblood, K. N. (1988). J. Phys. Chem. 92, 856-867.

Flaig, R., Koritsánszky, T. S., Dittrich, B., Wagner, A. \& Luger, P. (2002). J. Am. Chem. Soc. 124, 3407-3417.

Frisch, M. J. et al. (1995). Gaussian 94, revision B3. Gaussian Inc., Pittsburgh, Pennsylvania, USA.

Guillot, B., Jelsch, C., Podjarny, A. \& Lecomte, C. (2008). Acta Cryst. D64, 567-588.

Guillot, B., Viry, L., Guillot, R., Lecomte, C. \& Jelsch, C. (2001). J. Appl. Cryst. 34, 214-223.

Hammersley, A. P., Svensson, S. O. \& Thompson, A. (1994). Nucl. Instrum. Methods Phys. Res. A, 346, 312-321.

Hansen, N. K. \& Coppens, P. (1978). Acta Cryst. A34, 909-921.

Hartmann, K. (2003). Doktorarbeit. Zentrum für Kinderheilkunde und Jugendmedizin der Albert-Ludwigs-Universität Freiburg im Breisgau, Germany.

Hirshfeld, F. L. (1976). Acta Cryst. A32, 239-244.

Housset, D., Benabicha, F., Pichon-Pesme, V., Jelsch, C., Maierhofer, A., David, S., Fontecilla-Camps, J. C. \& Lecomte, C. (2000). Acta Cryst. D56, 151-160.

Hübschle, C. B., Luger, P. \& Dittrich, B. (2007). J. Appl. Cryst. 40, 623-627.

Husák, M., Kratochvíl, B. \& Císarová, I. (2000). Collect. Czech. Chem. Commun. 65, 1950-1958.

Husák, M., Kratochvíl, B., Jegorov, A., Mater'ha, V., Stuchlík, M. \& Andryásek, T. (1996). Z. Kristallogr. 211, 313-318.

Jelsch, C., Pichon-Pesme, V., Lecomte, C. \& Aubry, A. (1998). Acta Cryst. D54, 1306-1318.

Jelsch, C., Teeter, M. M., Lamzin, V., Pichon-Pesme, V., Blessing, R. H. \& Lecomte, C. (2000). Proc. Natl Acad. Sci. USA, 97, 31713176.

Johnas, S. K. J., Morgenroth, W. \& Weckert, E. (2006). Jahresber. HASYLAB, DESY, pp. 325-328.

Johnson, C. K. \& Levy, H. A. (1974). International Tables for X-ray Crystallography, Vol. IV, edited by J. A. Ibers \& W. C. Hamilton, pp. 311-335. Birmingham: The Kynoch Press.

Kabsch, W. (1993). J. Appl. Cryst. 26, 795-800.
Kalinowski, R., Dittrich, B., Hübschle, C. B., Paulmann, C. \& Luger, P. (2007). Acta Cryst. B63, 753-767.

Kallen, J., Sedrani, R., Zenke, G. \& Wagner, J. (2005). J. Biol. Chem. 280, 21965-21971.

Kendal, M. G. \& Stuart, A. (1958). The Advanced Theory of Statistics. London: Griffin.

Knott, R. B., Capel, M., Hansen, S. \& Handschumacher, R. E. (1995). J. Appl. Cryst. 28, 546-552.

Knott, R. B., Schefer, J. \& Schoenborn, B. P. (1990). Acta Cryst. C46, $1528-1533$

Koritsánszky, T. S. \& Coppens, P. (2001). Chem. Rev. 101, 15831627.

Kurki-Suonio, K. (1977). Isr. J. Chem. 16, 115-123.

Lecomte, C., Aubert, E., Legrand, V., Porcher, F., Pillet, S., Guillot, B. \& Jelsch, C. (2005). Z. Kristallogr. 220, 373-384.

Lecomte, C., Guillot, B., Muzet, N., Pichon-Pesme, V. \& Jelsch, C (2004). Cell. Mol. Life Sci. 61, 774-782.

Loosli, H.-R., Kessler, H., Oschkinat, H., Weber, H.-P., Petcher, T. J. \& Widmer, A. (1985). Helv. Chim. Acta, 68, 682-704.

Lüllmann, H., Mohr, K. \& Wehling, M. (2003). Pharmakologie und Toxikologie, 15 th ed. Stüttgart: Thieme.

Matta, C. F. \& Bader, R. F. W. (2003). Proteins, 52, 360-399.

Mebs, S., Messerschmidt, M. \& Luger, P. (2006). Z. Kristallogr. 221, 656-664

Meents, A., Dittrich, B., Johnas, S. K. J., Thome, V. \& Weckert, E. F. (2008). Acta Cryst. B64, 42-49.

Mikol, V. \& Duc, D. (1994). Acta Cryst. D50, 543-549.

Mikol, V., Kallen, J., Pflügl, G. \& Walkinshaw, M. D. (1993). J. Mol. Biol. 234, 1119-1130.

Moy, J. P., Hammersley, A. P., Svensson, S. O., Thompson, A., Brown, K., Claustre, L., Gonzalez, A. \& McSweeney, S. (1996). J. Synchrotron Rad. 3, 1-5.

Novartis (1989). Deutsches Patent DE 3843054 C2.

Petcher, T. J., Weber, H.-P. \& Rüegger, A. (1976). Helv. Chim. Acta, 59, 1480-1489.

Petrova, T. \& Podjarny, A. (2004). Rep. Prog. Phys. 67, 1565-1605.

Pflügl, G., Kallen, J., Schirmer, T., Jansonius, J. N., Zurini, M. G. \& Walkinshaw, M. D. (1993). Nature (London), 361, 91-94.

Pohl, E., Herbst-Irmer, R. \& Sheldrick, G. M. (1995). Helv. Chim. Acta, 78, 355-366.

Rödel, E., Messerschmidt, M., Dittrich, B. \& Luger, P. (2006). Org. Biomol. Chem. 4, 475-481.

Rosenfield, R. E., Trueblood, K. N. \& Dunitz, J. D. (1978). Acta Cryst. A34, 828-829.

Rüegger, A., Kuhn, M., Lichti, H., Loosli, H. R., Huguenin, R., Quiquerez, C. \& von Wartburg, A. (1976). Helv. Chim. Acta, 59, 1075-1092.

Schmidt, A. \& Lamzin, V. S. (2002). Curr. Opin. Struct. Biol. 12, 698-703.

Sheldrick, G. M. (2008). Acta Cryst. A64, 112-122.

Spitzfaden, C., Weber, H.-P., Braun, W., Kallen, J., Wider, G., Widmer, H., Walkinshaw, M. D. \& Wüthrich, K. (1992). FEBS Lett. 300, 291300 .

Sykes, P. (1988). Reaktionsmechanismen der Organischen Chemie. Weinheim: VCH Verlagsgesellschaft.

Thériault, Y., Logan, T. M., Meadows, R., Yu, L., Olejniczak, E. T., Holzmann, T. F., Simmer, R. L. \& Fesik, S. W. (1993). Nature (London), 361, 88-91.

Volkov, A., Abramov, Y., Coppens, P. \& Gatti, C. (2000). Acta Cryst. A56, 332-339.

Volkov, A., Li, X., Koritsánszky, T. S. \& Coppens, P. (2004). J. Phys. Chem. A, 108, 4283-4300.

Volkov, A., Macchi, P., Farrugia, L. J., Gatti, C., Mallinson, P. R., Richter, T. \& Koritsánszky, T. S. (2006). XD2006. A Computer Program Package for Multipole Refinement, Topological Analysis of Charge Densities and Evaluation of Intermolecular Energies from Experimental or Theoretical Structure Factors. http:// xd.chem.buffalo.edu/ 
Volkov, A., Messerschmidt, M. \& Coppens, P. (2007). Acta Cryst. D63, 160-170.

Vrielink, A. \& Sampson, N. (2003). Curr. Opin. Struct. Biol. 13, 709-715.

Watkin, D. (1994). Acta Cryst. A50, 411-437.

Weber, C., Wider, G., von Freyberg, B., Traber, R., Braun, W., Widmer, H. \& Wüthrich, K. (1991). Biochemistry, 30, 6563-6574.
Wenger, R. M., France, J., Bovermann, G., Walliser, L., Widmer, A. \& Widmer, H. (1994). FEBS Lett. 340, 255-259.

Wu, G., Rodrigues, B. L. \& Coppens, P. (2002). J. Appl. Cryst. 35, 356-359.

Zaleski, J., Wu, G. \& Coppens, P. (1998). J. Appl. Cryst. 31, 302-304.

Zarychta, B., Pichon-Pesme, V., Guillot, B., Lecomte, C. \& Jelsch, C. (2007). Acta Cryst. A63, 108-125. 